\title{
Les commis voyageurs, acteurs et témoins de la grande transformation
}

\author{
Arnaud BARTOLOMEI \\ Claire LEMERCIER \\ Silvia MARZAGALLI
}

Tout consommateur d'aujourd'hui a probablement déjà expérimenté le doute de celui qui conclut un achat en ligne, d'un bien ou d'une prestation d'une valeur significative, auprès d'une entreprise inconnue et plus ou moins totalement dématérialisée. Connue uniquement à travers son interface, cette entreprise à laquelle on vient de fournir un titre de paiement existe-t-elle réellement ? Est-elle fiable ? Tiendra-t-elle ses engagements et quels sont les recours possibles en cas de malversation ? Force est cependant de constater que de telles incertitudes n'ont pas empêché l'essor spectaculaire du commerce en ligne au cours de la dernière décen-nie, auprès des consommateurs comme des entreprises ${ }^{1}$.

Replacé dans un temps long, celui qui court de la renaissance du commerce à distance en Europe à la fin du haut Moyen Âge jusqu'à nos jours, ce succès d'un commerce impersonnel, dit " virtuel ", peut apparaître comme l'ultime étape d'un processus de transformation de l'échange marchand qui l'aurait peu à peu émancipé de toutes les contraintes sociales, cultu-relles ou tout simplement physiques. Du troc pratiqué par des marchands itinérants jusqu'aux échanges exclusifs développés dans le cadre sécurisant des diasporas marchandes, puis des opérations menées, depuis son comptoir, par le négociant au sein d'un réseau de correspon-dants patiemment construit et scrupuleusement entretenu jusqu'à la conquête de vastes mar-chés au moyen de la publicité et du marketing, l'histoire du commerce à distance au cours du dernier millénaire serait alors celle d'un cheminement ininterrompu vers une pratique toujours plus dématérialisée, dépersonnalisée et détachée des supports traditionnels de la confiance que sont la connaissance personnelle, la familiarité ou l'appartenance à un même groupe.

\footnotetext{
${ }^{1}$ Cf. P. Volle (dir.), " Le commerce électronique : évolution ou révolution ? ", Entreprises et Histoire, $n^{\circ} 64$, sep- $^{\circ}$ tembre 2011, p. 167-182.
} 
L'histoire d'un tel désencastrement de l'échange commercial reste largement à écrire ; mais elle est sans doute l'un des symptômes les plus significatifs de ce que les chercheurs en sciences sociales, à la suite de Karl Polanyi, ont pris l'habitude d'appeler la Grande Transformation ${ }^{2}$. En effet, le désencastrement du commerce peut être interprété à la fois comme une conséquence de l'émergence d'une société plus libérale et moins communautaire et comme un élément moteur de cette évolution puisqu'il a notamment accompagné la standardisation - et l'essor - de la production industrielle et le développement de la société de consommation. Si un tel « grand récit » peut sembler trop linéaire, sa vertu heuristique est indéniable. Il faudra bien sûr le nuancer, lui redonner de la complexité ; mais il permet de structurer un questionnement sur des pratiques du commerce finalement encore mal connues pour la période qui va du XVIII ${ }^{\mathrm{e}}$ siècle à nos jours. S'il faut les étudier empiriquement, dans leur diversité sectorielle et territoriale, ce n'est donc pas seulement pour elles-mêmes, mais aussi pour comprendre le rôle qu'ont joué leurs transformations dans la formation des sociétés actuelles. Le dossier réuni ici autour de la figure du commis voyageur, répond à ces deux objectifs puisque, à partir de l'histoire d'un métier du commerce encore mal identifié, il nous offre un observatoire saisissant sur les mécanismes qui ont conduit à la mise en place de notre monde actuel.

Les commis voyageurs, jusqu'ici ignorés de la recherche en sciences sociales, sont apparus précisément durant l'époque à laquelle on fait habituellement remonter les prémices de la Grande Transformation - la seconde moitié du XVIII ${ }^{\mathrm{e}}$ siècle - et ils en furent des acteurs centraux tout autant que des témoins privilégiés. Leur histoire permet donc de réfléchir à la pertinence de ce grand récit, d'en préciser la chronologie et surtout d'en mieux comprendre les quatre principaux rouages : la constitution de vastes marchés unifiés en Europe et en Amérique, la rationalisation de l'organisation des firmes, la formation de la classe moyenne et le mouvement de dépersonnalisation des relations économiques. C'est donc dans le dessein de participer à l'émergence d'une historiographie des pratiques commerciales contemporaines tout en contribuant à la réflexion sur ces quatre dynamiques constitutives de la Grande Transformation qu'a été conçu le présent numéro d'Entreprises et Histoire.

\section{LE COMMIS VOYAGEUR : UNE FIGURE IGNORÉE DU MONDE CONTEMPORAIN}

Le commis voyageur n'est pas réputé pour sa discrétion ou sa transparence : ce sont plutôt sa faconde, voire ses plaisanteries grossières, qui le caractérisent dans notre fonds culturel commun. Mais, au-delà de ce cliché, force est de constater que nous savons peu de choses sur les commis voyageurs et sur leurs descendants, les représentants de commerce, appelés aussi VRP (pour voyageurs-représentants-placiers) ou agents commerciaux en France, traveling salesmen ou traveling drummers dans le monde anglophone. Certes, ce sont des métiers de la vente, associés à l'idée d'un démarchage itinérant de clients. Mais qui saurait préciser si ces « voyageurs de commerce » agissent de façon indépendante ou pour le compte de firmes, s'ils démarchent des particuliers ou des entreprises, de quelle manière ils sont rémunérés ? Qui serait en mesure de disserter sur leur utilité réelle ou sur les raisons qui expliquent leur résis-

\footnotetext{
${ }^{2}$ K. Polanyi, La grande transformation. Aux origines politiques et économiques de notre temps, Paris, Gallimard, 1983 (édition originale : 1944). Pour un aperçu des débats récents sur ce livre, cf. A. Hatchuel, O. Favereau, F. Aggeri (dir.), L'activité marchande sans le marché ?, Paris, Presses de 1'Ecole des Mines, 2010. Nous employons ici l'expression «Grande Transformation » pour désigner le phénomène d'autonomisation de la sphère économique et non pour désigner comme K. Polanyi le faisait, le processus de réencastrement des économies qui se serait manifesté dans la première moitié du $\mathrm{XX}^{\mathrm{e}}$ siècle.
} 
tance face à l'essor du marketing et des nouvelles technologies de la communication ? Qui sait enfin combien ils sont ou quelles organisations collectives les représentent ? C'est dire l'ampleur de notre ignorance sur des métiers qui, s'ils sont fréquemment cités dans la langue courante, sont rarement évoqués pour ce qu'ils sont réellement, comme l'atteste suffisamment l'interrogation des bases de données textuelles accessibles en ligne.

\section{Un topos plus qu'une figure littéraire}

C'est d'abord à la pièce Mort d'un commis voyageur (Death of a Salesman, 1949) d'Arthur Miller, montée des centaines de fois sur toutes les scènes du monde et adaptée pour le cinéma, que renvoie aujourd'hui l'expression « commis voyageur ». Le célèbre dramaturge américain décrit, à travers la déchéance de son protagoniste, un représentant de commerce fatigué par une vie de labeur mal considéré et obsédé par l'espoir d'une réussite sociale qui lui échappe et qu'il reporte sur ses fils, les ambiguïtés d'un rêve américain dont il dénonce finalement les faux-semblants. Mais, à l'exception de cet anti-héros, le représentant de commerce est presque totalement absent de la littérature du $\mathrm{XX}^{\mathrm{e}}$ siècle. Le constat est différent pour le siècle précédent, en revanche, au moins en ce qui concerne la France, comme l'atteste la recherche effectuée dans la base de données textuelles Frantext ${ }^{3}$.

\begin{tabular}{|l|l|}
\hline Expression & Nombre d'occurrences total \\
\hline Marchand/s & 10812 \\
\hline Commerçant/s & 2045 \\
\hline Négociant/s & 1715 \\
\hline Boulanger/s & 1561 \\
\hline Colporteur/s & 445 \\
\hline Boutiquier/s & 413 \\
\hline Commis voyageur/s & 358 (dont 276 avant 1914 et 82 après) \\
\hline Voyageur/s de commerce & 85 (dont 13 avant 1914 et 72 après) \\
\hline Représentant/s de commerce & 42 (dont 6 avant 1914 et 36 après) \\
\hline
\end{tabular}

Tableau 1 : Les commis voyageurs et les autres métiers du commerce dans la base de données Frantext

Ces résultats, s'ils confirment la position en retrait qu'occupent les commis dans l'imaginaire français par rapport à d'autres métiers du commerce, montrent aussi que la littérature du $\mathrm{XIX}^{\mathrm{e}}$ siècle les a moins ignorés que celle du $\mathrm{XX}^{\mathrm{e}}$. Mais quelle place leur a-t-elle donnée ? Sauf dans L'Illustre Gaudissart de Balzac, c'est avant tout comme des éléments de décor ou d'anecdote que sont utilisés les commis voyageurs, moins intéressants pour eux-mêmes que

\footnotetext{
${ }^{3}$ Recherche effectuée le 6 septembre 2010 sur le site du laboratoire Analyse et traitement informatique de la langue française (ATILF) : http://www.atilf.fr/. Frantext réunit un corpus numérisé de plus de 4000 textes littéraires français. Les deux orthographes usuelles « commis voyageur » et « commis-voyageur » ont été prises en compte.
} 
par ce que le nom de leur métier permet d'évoquer en raccourci : le voyage, mais aussi, voire surtout, une figure quelque peu inculte de la classe moyenne et de son matérialisme petit-bourgeois. Dans les récits de voyage, alors très en vogue, ils servent ainsi de simple faire-valoir anonyme à des narrateurs désireux de se mettre en valeur :

« En parlant de bandits, M. Croiset nous raconta qu'en traversant l'Italie il avait bien pensé n'en jamais sortir. Il voyageait de nuit avec le courrier et un commis de négociant, quand, à Cisterno, dans les Marais Pontins, il fut éveillé par plusieurs coups de fusils. La voiture fut de suite assaillie par plusieurs bandits, et les trois voyageurs eurent ordre de descendre. On se mit à fouiller leurs effets, tandis qu'on les tenait tous les trois sous les stylets. Le commis voyageur en reçut un coup à la gorge qui lui a placé la tête de côté pour toute la vie. Croiset ne fut pas blessé $[\ldots] »$.

Ailleurs, ce sont les allusions dépréciatives - plus même qu'anonymes, impersonnelles qui abondent, même si quelques auteurs insistent sur la faconde et l'entrain associés au métier. Un héros de George Sand constate amèrement : " Je crus comprendre qu'à la platitude de mon expression elle me prenait pour un commis voyageur $»^{5}$. Le père de Flaubert conseille à son fils, sur le point de partir au Levant, de ne pas voyager " en épicier ou en commis voyageur », c'est-à-dire d'être sensible aux beautés des pays traversés, contrairement à ceux qui ne veulent que vendre. Et le Journal des Goncourt ne manque jamais une occasion d'épingler la lourdeur, voire la grossièreté, des commis : « une grosse gaîté lourde de commis-voyageur », « le voltairianisme d'un commis-voyageur »(1863), "c'est un verbeux, qui me rappelle la bruyance et le commun des paroles d'un commis-voyageur à un dîner d'auberge du temps des diligences » (1890).

En revanche, aucune occurrence n'apparaît avant 1831 - alors que, les articles ici réunis le confirment, le métier existe alors depuis plusieurs décennies - et, au XX $\mathrm{XX}^{\mathrm{e}}$ siècle, l'expression " commis voyageur ", qui, il est vrai, tombe en désuétude face à " voyageur de commerce » (qui apparaît en 1866 dans Frantext) et « représentant de commerce » (1881), tend à perdre toute consistance sociale pour se réduire à un simple stéréotype, que l'on emploie sur un air entendu et allusif : de Proust à Mendès France, on parle alors de " plaisanterie ", d' ' esprit » ou de « jeu de mots » de « commis voyageurs ». Grâce à Balzac et à d'autres, c'est donc dans les années 1840 que le commis voyageur connaît en France son pic de notoriété en tant que figure littéraire : certainement parce que le métier, qui a pris suffisamment d'importance pour être individualisé, se trouve alors associé à une certaine idée de la modernité ; au contraire, il se banalise ensuite au point de devenir invisible.

\section{Un inconnu pour les sciences sociales}

Si la présence des commis voyageurs dans la littérature fournit un premier indice quant à leur rapport à la modernité, elle reste finalement limitée. Elle est tout de même plus forte qu'en sciences sociales, où l'emploi du nom du métier se limite en général à un usage strictement métaphorique ${ }^{6}$. On parle ainsi de «commis voyageur» de la République, du PCF, de

\footnotetext{
${ }^{4}$ A. Creuzé de Lesser, Voyage en Italie et en Sicile fait en 1801 et 1802, Paris, Didot, 1806, p. 103.

${ }^{5}$ G. Sand, Lettres d'un voyageur, Paris, Michel Lévy frères, 1857, p. 9.

${ }^{6}$ Nos constats, dans cette partie, se fondent notamment sur une recherche effectuée à partir du moteur Google Scholar ainsi que sur les portails francophones Cairn et Revues.org, en plusieurs étapes en juin et octobre 2010.
} 
l'art royal, du nationalisme algérien ou de la poésie, comme si ce que recouvre l'expression (voyager pour convaincre ?) était évident. Seuls les juristes, auxquels la complexité du statut de ces métiers n'a pas échappé, font exception et représentent une portion significative de la partie numérisée de l'iceberg bibliographique ${ }^{7}$. Rien, en revanche, en France du moins, chez les chercheurs en gestion qui se sont, il est vrai, peu intéressés, d'une manière plus générale, aux métiers de la vente ${ }^{8}$. Peu de choses également chez les sociologues du travail, qui ont eux aussi délaissé l'étude de ces professions jusqu'à une période très récente ${ }^{9}$; et lorsqu'une spécialiste de la famille et de l'héritage fait du commis voyageur un idéal-type, c'est exclusivement en référence à la pièce d'Arthur Miller ${ }^{10}$.

Les historiens leur ont aussi réservé la portion congrue dans leurs travaux. Les quelques études qui existent en français sont de qualité mais éparpillées et peu accessibles. Nous n'avons pu trouver que deux articles réellement consacrés à des voyageurs de commerce : comme un travail plus récent, encore inédit, de Robert Darnton, ils suivent un seul voyage à partir d'une source particulièrement riche, sans proposer de généralisation sur l'activité ${ }^{11}$. D'autres articles évoquent, de façon périphérique, le rôle des commis voyageurs dans les circuits du commerce international ${ }^{12}$. Mais rares sont les études de cas qui analysent systématiquement leur rôle dans une entreprise ou dans un secteur industriel : seuls les passages qui leur sont consacrés dans les thèses de Claude Fohlen, de Marc de Ferrière et surtout de Gérard Gayot font exception ${ }^{13}$. Or ces quelques références éparses ne constituent pas un corpus de connaissances suffisant pour avoir été intégré aux grandes synthèses d'histoire économique. Celles-ci accordent d'ailleurs peu d'importance au secteur et aux fonctions commerciales, en tout cas pour la période contemporaine : ainsi, Pierre Léon ne consacre qu'une dizaine de lignes aux voyageurs de commerce dans sa contribution au volume III de l'Histoire écono-

\footnotetext{
${ }^{7}$ En témoigne le document commenté dans le présent numéro de la revue Entreprises et Histoire.

${ }^{8}$ Parmi plusieurs publications d'Isabelle Barth, voir par exemple « Regard prospectif sur les métiers commerciaux. Du vendeur ambassadeur au core seller », Management \& Avenir, $\mathrm{n}^{\circ} 25$, juin 2009, p. 371-385.

${ }^{9}$ Constat formulé notamment lors de la journée d'étude de l'UMR Cultures et sociétés urbaines « La stratification sociale au travail : le cas des activités commerciales » le 18 janvier 2010 (http://calenda.revues.org/nouvelle15001.html - référence du $1^{\mathrm{er}}$ octobre 2010) qui a permis de discuter le travail de Jérôme Greffion sur les visiteurs médicaux ; Cédric Lomba s'intéresse plus largement aux pratiques de vente dans la pharmacie. La thèse en cours de Lise Bernard, elle, porte sur la place des agents immobiliers dans la stratification sociale.

${ }^{10}$ A. Gotman, «L'héritier et le commis voyageur. Transmission et héritage de la maison de famille », in M. Segalen (dir.), Jeux de familles, Paris, CNRS Éditions, 2002.

${ }^{11} \mathrm{~S}$. de Montenay, « Les tribulations d'un voyageur de commerce à la fin de l'Ancien Régime, ou de l'inconvénient de ne point savoir parler allemand en Allemagne », Annales de Bourgogne, t. XXXIX, 1967, p. 197-235 et J.-J. Chevalier, "La tournée française d'un représentant choletais en mouchoirs (1849) », in Le mouchoir dans tous ses états. Actes du colloque international, Cholet, Musée du textile choletais, 1997, p. 49-63. On peut écouter en ligne R. Darnton, «Étude du journal d'un commis voyageur », in « L'édition et la librairie sous l'Ancien Régime : la science de la contrefaçon ", Archives audiovisuelles de la recherche, 26 janvier 2004, http://www.archivesaudiovisuelles.fr/190 (consultation du 11 octobre 2010).

${ }^{12}$ Voir par exemple J.-L. Regemorter. « Le mythe génois en mer Noire : la France, la Russie et le commerce d'Asie par la route de Géorgie (1821-1881) », Annales ESC, 19, 3, 1964, p. 492-521 et C. Beaud, "L'innovation des établissements Schneider (1837-1960) », Histoire, économie et société, 14, 3, 1995, p. 501-518.

${ }^{13}$ C. Fohlen, Une affaire de famille au XIX siècle, Méquillet-Noblot et Cie, Paris, Armand Colin, 1955, repris dans C. Fohlen, L'industrie textile au temps du Second Empire, Paris, Plon, 1956, p. 151-152 ; M. de Ferrière, Christofle : deux siècles d'aventure industrielle (1793-1993), Paris, Le Monde Éditions, 1995, p. 127-136 ; G. Gayot, Les draps de Sedan, 1646-1870, Paris, Éditions de l'EHESS, 1998, notamment p. 399-405.
} 
mique et sociale de la France, significativement intitulé « L'ère industrielle » ${ }^{14}$. L'Histoire générale du travail propose bien quelques pages sur les colporteurs et les voyageurs de commerce, mais le traitement est anecdotique plus que réellement analytique, même si la modernité de ces métiers est bien mise en exergue ${ }^{15}$. Enfin, on ne peut que s'étonner de l'absence des voyageurs de commerce dans les ouvrages importants qui ont récemment été consacrés à l'histoire culturelle du voyage, pourtant attentifs au cas du voyage d'affaires ${ }^{16}$. Il a donc fallu attendre l'accent mis délibérément par Patrick Verley sur le poids de la demande dans la révolution industrielle pour lire un véritable essai de synthèse intégrant à sa juste place le métier de commis voyageur ${ }^{17}$.

La situation ailleurs en Europe, bien que l'enquête que nous avons pu mener sur la question soit loin d'être exhaustive, semble à peu de choses près similaire ${ }^{18}$. Elle est en revanche très différente aux États-Unis, où les représentants de commerce ont trouvé leur historien en la personne de Walter A. Friedman ${ }^{19}$. Son ouvrage de synthèse s'appuie tant sur des sources imprimées (manuels, guides du voyageur, presse spécialisée) que sur de nombreux travaux déjà publiés ${ }^{20}$ pour revenir sur la genèse du métier, son expansion pendant la forte croissance industrielle des années 1870-1900 - le nombre de traveling drummers passe ainsi de 7000 dans le recensement de 1870 à 60000 en 1890 -, puis les transformations du $\mathrm{XX}^{\mathrm{e}}$ siècle. Il évoque aussi l'image d'une activité qui suscita autant de fascination - en raison notamment de la maîtrise de l'art de la persuasion qu'elle supposait - que d'hostilité - chez les marchands sédentaires en particulier.

Comment expliquer cette plus grande attention portée par l'historiographie américaine à des professions dont l'étude est négligée en Europe ? Découle-t-elle d'un plus grand rôle qu'elles auraient joué dans le système commercial outre-Atlantique ? Ou encore du fait

\footnotetext{
${ }^{14}$ F. Braudel et E. Labrousse (dir.), Histoire économique et sociale de la France, t. III : L'avènement de l'ère industrielle (1789-1880), Paris, PUF, 1993 ( $1^{\text {re }}$ éd. : 1976), p. 287-288. Le traitement de la question n'est pas plus précis chez J. Lacour-Gayet, Histoire du commerce, t. IV et V, Paris, SPID, 1952.

${ }^{15}$ C. Fohlen et F. Bédarida (dir.), Histoire générale du travail, t. III, Paris, Nouvelle Librairie de France, 1996 ( $1^{\mathrm{re}}$ éd. : 1960), p. 17-32 et 225-226.

${ }^{16}$ Les commis voyageurs sont presque totalement absents de F. Angiolini et D. Roche (dir.), Cultures et formations négociantes dans l'Europe moderne, Paris, Éditions de l'EHESS, 1995 ; D. Roche, Humeurs vagabondes. De la circulation des hommes et de l'utilité des voyages, Paris, Fayard, 2003 ; A. Burkardt (dir.), Commerce, voyage et expérience religieuse, $X V I^{e}$-XVIII ${ }^{e}$ siècles, Rennes, Presses Universitaires de Rennes, 2007.

${ }^{17}$ P. Verley, L'échelle du monde. Essai sur l'industrialisation de l'Occident, Paris, Gallimard, 1997, p. 260-274.

${ }^{18}$ Stanley Chapman n'accorde, par exemple, que peu d'attention aux commis voyageurs dans sa synthèse consacrée pourtant à la révolution commerciale en Angleterre aux $\mathrm{XVIII}^{\mathrm{e}}$ et $\mathrm{XIX}^{\mathrm{e}}$ siècles et tout particulièrement aux relations entre grossistes et détaillants (S. Chapman, Merchant Enterprise in Britain from the Industrial Revolution to World War I, Cambridge-New York, Cambrige University Press, 1992). Nous confessons nos lacunes du côté de 1'historiographie germanophone.

${ }^{19}$ W. A. Friedman, Birth of a Salesman. The Transformation of Selling in America, Cambridge (Mass.), Harvard University Press, 2004. Voir aussi son article de postface, du présent numéro.

${ }^{20}$ Principalement J. R. Dolan, The Yankee Peddlers of Early America : An Affectionate History of Life and Commerce in the Developing Colonies and the Young Republic, New York, C. N. Potter, 1964, S. C. Hollander, " Nineteenth Century Anti-Drummer Legislation in the United States », Business History Review, 38, Winter 1964, p. 479-500, D. Jaffee, " Peddlers of Progress and the Transformation of the Rural North, 1760-1860 », Journal of American History, September 1991, p. 511-535, T. B. Spears, One Hundred Years on the Road : The Traveling Salesman in American Culture, New York, Yale University Press, 1997. La question des voyageurs de commerce est par ailleurs largement traitée dans des ouvrages de synthèse consacrés à la révolution commerciale (A. D. Chandler, La main visible des managers. Une analyse historique, Paris, Economica, 1988 (édition originale : 1977)) ou à la formation du marché intérieur américain (S. Strasser, Satisfaction Guaranteed: The Making of the American Mass Market, New York, Pantheon Books, 1989).
} 
qu'elles auraient laissé plus de traces dans les fonds d'archives ? La lecture des contributions réunies ici amène à balayer ces deux hypothèses. En Europe aussi, c'est par milliers, voire dizaines de milliers, que les voyageurs de commerce sillonnèrent les routes, et ce dès le $\mathrm{XVIII}^{\mathrm{e}}$ siècle : ainsi, Chardon, un commis voyageur en vins de Bourgogne qui prospecte l'Allemagne en 1784, ne cesse de se plaindre de l'abondante concurrence avec laquelle il doit composer et évoque dans sa correspondance un aubergiste de Francfort qui aurait accueilli jusqu'à 62 voyageurs de commerce en une seule année ${ }^{21}$; Gérard Gayot, pour sa part, a dénombré 160 commis voyageurs au service des 30 draperies de Sedan en $1778^{22}$. Alors que l'historiographie associait souvent ses brèves mentions du voyageur de commerce à la mise en place du réseau ferré en Europe, après $1840^{23}$, faisant donc dépendre son activité d'une industrialisation déjà bien entamée, le constat de cette forte présence un bon demi-siècle plus tôt conduit à inverser la perspective et à admettre au contraire la grande banalité de la figure du commis voyageur dans l'Europe des années 1780.

En outre, les sources ne font pas défaut. Dans certaines archives privées, on trouve les feuilles de route des voyageurs, leurs correspondances - professionnelle ou privée - ou encore leurs carnets et leurs comptes ${ }^{24}$. L'exploitation de ces fonds, encore très rarement indexés, reste un travail de longue haleine, mais crucial pour comprendre notamment la place des représentants dans les stratégies des entreprises. Cela dit, nombre d'autres sources peuvent être inventées : rapports d'inspecteurs des manufactures (déjà utilisés par Gérard Gayot) ou de consuls ; sources classiques de l'histoire sociale (actes d'état civil, recensements, actes notariés, etc.), ou sources plus spécifiques comme les fonds de passeports, dans l'ensemble bien conservés à partir du XVIII ${ }^{\mathrm{e}}$ siècle, qui devraient permettre d'apprécier le poids des voyageurs de commerce dans l'ensemble des circulations marchandes et de dater les manières de désigner cette activité ; et surtout des imprimés très divers, parfois numérisés, qui permettent d'aborder, par exemple, les débats soulevés par le statut juridique des voyageurs, leur organisation en syndicats ou encore leur image dans la littérature et dans la presse ${ }^{25}$.

Nous avons pu repérer ainsi, grâce à une recherche en ligne sur leur texte intégral, des documents riches en information sur le métier, dont les titres n'auguraient pourtant pas du contenu en la matière et qui démontrent la variété des recherches qui pourraient être entreprises. Dans un manuel de conversation franco-italien publié en 1814, le chapitre consacré à la visite d'un voyageur de commerce lyonnais chez un marchand-détaillant d'une ville italienne résume en quelques lignes sa mission : élargir la clientèle de son commettant dans des régions où ce dernier n'a pas de réputation, en présentant directement ses productions aux commerçants locaux ${ }^{26}$. Ce type de littérature pratique nous informe autant par son contenu que par son existence même : les voyageurs de commerce pouvaient, au XIX ${ }^{\mathrm{e}}$ siècle, utiliser des guides comprenant des informations pratiques sur les transports ou les modalités d'hébergement,

\footnotetext{
${ }^{21}$ S. de Montenay, « Les tribulations ... », art. cit., p. 235.

${ }^{22}$ G. Gayot, Les draps de Sedan ..., op. cit., p. 405.

${ }^{23}$ C'est le cas notamment dans J. Lacour-Gayet, Histoire du commerce, op. cit., et F. Braudel et E. Labrousse (dir.), Histoire économique et sociale de la France, op. cit., t. III, p. 287.

${ }^{24}$ Plusieurs articles du présent numéro utilisent de telles sources privées : cf. les contributions d'A. Orlandi, F. Bayard, A. Bartolomei, B. Musset, L. Panariti, F. Perron, S. Vabre et J. Villain.

${ }^{25}$ Cf. les contributions au présent numéro de D. Andreozzi, S. A. Marin, J.-P. Barrière, R. Rossfeld.

${ }^{26}$ P. L. Costantini, Dialoghi italiani e francesi all'uso delle due nazioni, Paris, Amand Koening, 1814 (des extraits de cet ouvrage sont présentés et commentés dans la rubrique «Clin d'oeil » du présent numéro d'Entreprises et Histoire).
} 
mais aussi des listes des boutiquiers de chaque ville, qui leur permettaient de planifier leurs séjours. Les manuels destinés aux marchands, mine bien connue des modernistes mais trop ignorée en histoire contemporaine ${ }^{27}$, racontent un autre versant de l'histoire des voyageurs de commerce. Dans celui publié par Louis Deplanque en 1844, une dizaine de pages sont consacrées aux écritures correspondant aux diverses missions qu'un commis voyageur est susceptible d'accomplir : vente de marchandises, achat, recouvrement d'effets, etc. ${ }^{28}$. Ce texte nous révèle donc, derrière l'image omniprésente de la vente, les multiples fonctions que recouvrait en fait le métier. Un compte de calcul de la rentabilité du voyage est également proposé, qui permet de réfléchir sur la manière dont les contemporains eux-mêmes se représentaient la rationalité de l'emploi de voyageurs.

\section{COMMIS VOYAGEURS ET MODERNITÉ}

La réflexion collective qui a été entreprise lors du colloque organisé par le Centre de la Méditerranée Moderne et Contemporaine (CMMC) de Nice en $2010^{29}$, et que prolonge la présente publication, ne visait pourtant pas seulement à combler un vide historiographique, ni à juxtaposer de simples études de cas, même si celles-ci battent déjà en brèche nombre d'idées reçues. À travers l'étude d'un métier, nous avons souhaité contribuer à des débats plus larges sur l'intermédiation marchande, ses formes, ses logiques et le rôle que ses transformations ont joué dans la dynamique globale des sociétés occidentales. Comme Gérard Gayot l'avait pressenti dans les pages lumineuses qu'il a consacrées à ceux qu'il appelait les « voyageurs intelligents », leur émergence a en effet partie liée avec au moins quatre grandes transformations qui fondent notre modernité : la révolution industrielle - puisque c'est bien pour élargir leurs marchés, en contournant le monopole d'exportation des commissionnaires parisiens, et soutenir ainsi leur activité productive, que les manufacturiers de Sedan se sont mis à employer des commis voyageurs - ; la naissance de la grande entreprise et de son organisation rationalisée - à la fois salariés et intéressés aux ventes, les commis se voient fournir des outils communs, comme les catalogues et les circulaires, qui bornent leur autonomie - ; l'émergence d'une classe moyenne qu'évoque Gérard Gayot lorsqu'il remarque qu' « un petit monde tertiaire était en train de se former »; enfin, la dépersonnalisation ambiguë des relations commerciales puisque, s'ils sont censés démarcher pour leur firme une clientèle de partenaires encore inconnus, les commis sont le plus souvent introduits auprès de ces derniers par des lettres de recommandation et ont finalement pour fonction première l'incarnation des sociétés qui les emploient.

\section{La révolution industrielle}

Stanley Chapman aussi bien que Walter A. Friedman et Patrick Verley ont placé le processus de modernisation de l'intermédiation marchande - et de baisse de son coût - qui traverse de part en part les $\mathrm{XVIII}^{\mathrm{e}}, \mathrm{XIX}^{\mathrm{e}}$ et $\mathrm{XX}^{\mathrm{e}}$ siècles au cœur de la dynamique économique et

\footnotetext{
${ }^{27}$ Voir la collection des Ars Mercatoria. Handbücher und Traktate für den Gebrauch des Kaufmanns : 1470-1820. Eine analytische Bibliographie, éditée par P. Jeannin, J. Hoock et W. Kaiser (Paderborn, Munich et Vienne, Schöningh, 1991, pour le premier volume, 1993, pour le deuxième et 2001, pour le troisième).

${ }^{28}$ La tenue des livres en partie simple et en partie double, Paris, Dutertre, 1844.

${ }^{29}$ « Les figures de l'intermédiation marchande en Europe et dans le monde méditerranéen $\left(\mathrm{XVI}^{\mathrm{e}}-\mathrm{XXI}{ }^{\mathrm{e}}\right.$ siècles) : commis voyageurs et représentants de commerce », Université de Nice-Sophia Antipolis, 10-12 juin 2010.
} 
sociale majeure des sociétés européennes et américaines : la révolution industrielle. Pour eux, le processus d'innovation technologique et de croissance continue de la production que cette expression désigne a en effet été suscité par la formation d'un vaste marché de consommateurs, demandeurs de produits standardisés. Or ce qui aurait contribué à unifier et à élargir ce marché, plus que la hausse du niveau de vie - qui ne devient réelle qu'à l'extrême fín du $\mathrm{XIX}^{\mathrm{e}}$ siècle -, c'est la modernisation des réseaux de transport et de distribution. L'intensification et l'accélération des échanges terrestres et maritimes, la densification du réseau de boutiques ou encore l'émergence de nouveaux acteurs de l'intermédiation marchande, qui viennent concurrencer les anciens monopoles négociants, auraient été autant de facteurs permettant l'émergence sinon de la société de consommation, du moins d'une société de consommateurs - fondée sur des actes devenus banals pour un nombre toujours plus grand de contemporains, allant de l'achat d'un peigne ou d'un almanach à un colporteur au renouvellement régulier d'une garde-robe dans les boutiques urbaines. Or, si l'évolution des consommations a été bien documentée, à partir notamment des inventaires après décès, on commence à peine, malgré des travaux pionniers sur le colportage ou sur la boutique ${ }^{30}$, à mieux connaître les moyens concrets utilisés pour la vente, avant le temps des grands magasins puis de la publicité ; l'approvisionnement des détaillants et leur rôle dans l'évolution des goûts restent, quant à eux, des terres vierges pour la recherche.

De ce point de vue, le commis voyageur apparaît comme un des instruments employés par les fabricants de biens manufacturés pour contourner la coûteuse intermédiation proposée par les circuits négociants traditionnels et entrer directement en contact avec leur clientèle, s'informer sur ses goûts et ses habitudes et élargir ainsi leurs débouchés. Les études de cas proposées par Gérard Gayot et Jean-Joseph Chevalier s'inscrivent dans ce modèle interprétatif d'ensemble. Il faudrait cependant le discuter à l'aune des études menées aux États-Unis : les grossistes américains ont eux aussi été précurseurs dans l'emploi de représentants, et il serait donc réducteur de ne voir en ces derniers que des employés des grandes firmes industrielles. De même, en France, ce sont les négociants en vins qui semblent avoir joué un rôle pionnier ${ }^{31}$. Sortir de la monographie par des comparaisons s'avère donc nécessaire pour distinguer ce qui relève de stratégies propres à une firme, à un moment donné, de ce qui découle de la tendance d'ensemble : la reconfiguration des circuits commerciaux au profit des industriels. Il serait alors possible d'appuyer, ou de nuancer, l'idée selon laquelle le commis voyageur, incontestablement né de pratiques négociantes dans un monde négociant, serait finalement devenu le fossoyeur du négoce traditionnel.

Dans le champ de réflexion connexe de la géographie économique, la question de l'emploi des commis voyageurs, et plus généralement de l'efficacité des différentes infrastructures commerciales nationales - ou régionales, ou sectorielles ? - en concurrence sur le continent européen a aussi été posée pour expliquer la plus ou moins grande performance économique des territoires. Ainsi s'est forgée l'idée que le commis voyageur allemand aurait contribué à asseoir la suprématie de l'industrie de son pays sur ses rivales européennes ${ }^{32}$. Là encore, le

\footnotetext{
${ }^{30}$ M.-E. Chessel, Histoire de la consommation, Paris, La Découverte, 2012. Voir notamment L. Fontaine, Histoire du colportage en Europe (XV $-X I X^{e}$ siècle), Paris, Albin Michel, 1993 ; D. Roche, Histoire des choses banales. Naissance de la consommation dans les sociétés traditionnelles (XVII -XIX ${ }^{e}$ siècle), Paris, Fayard, 1997 ; N. Coquery (dir.), La Boutique et la ville. Commerces, commerçants, espaces et clientèles, $X V I^{e}-X X^{e}$ siècle, Tours, Centre d'histoire de la ville moderne et contemporaine/Publications de l’Université François Rabelais, 2000.

${ }^{31} \mathrm{Cf}$. les contributions de B. Musset et F. Perron au présent numéro.

${ }^{32} \mathrm{Cf}$. la contribution de S. A. Marin au présent numéro.
} 
commis voyageur apparaît comme un chaînon manquant - dont le rôle est supposé, mais mal connu - dans les explications de la révolution industrielle et de sa chronologie.

\section{La naissance de la grande entreprise}

Si le recours à des commis voyageurs par des industriels peut être vu comme un moyen pour eux de se passer des négociants, cela suppose qu'ils aient eu un plus grand intérêt à utiliser ceux-là que ceux-ci, en raison du moindre coût de leurs services, des avantages qu'ils leur offraient - en termes de retour d'informations sur les marchés, par exemple - et, plus généralement, du plus grand contrôle qu'il était possible d'exercer sur eux. D'où le caractère crucial que revêt l'étude du statut et du mode de rémunération des voyageurs - et la possibilité de contribuer, par ce biais, à des interrogations aussi décisives en économie et en gestion que celles qui portent sur la naissance de la grande firme intégrée, sa chronologie et sa rationalité propre $^{33}$. L'histoire des commis voyageurs est également importante pour celle du salariat, puisque leur cas - très ambigu - a donné lieu, en France, à une abondante jurisprudence contribuant à cerner cette notion toute nouvelle du XX $\mathrm{X}^{\mathrm{e}}$ siècle : ont ainsi été discutés, entre autres, leur droit à des congés, au repos dominical, les formes de leur rémunération, leur degré d'autonomie - les engagements pris par le commis obligent-ils son employeur ? -, ou encore la question de la patrimonialité de la clientèle, problème crucial en cas de rupture de contrat ${ }^{34}$.

Du point de vue des entreprises, c'est l'enjeu stratégique de l'internalisation de la force de vente qui est soulevé par l'emploi des commis voyageurs et par les conditions qui leur sont réservées. Les travaux de Ronald Coase et d'Oliver Williamson sur l'organisation des firmes et le rôle qu'elles ont joué dans la réduction de l'incertitude et des coûts de transaction dans le commerce à distance font ici référence ${ }^{35}$. Mais toute une lignée de recherches sur la « spécialisation flexible » insiste au contraire, en résonance avec les pratiques d'aujourd'hui en matière d'externalisation ou de "firme en réseau », sur l'efficacité potentielle de pratiques contraires $^{36}$. L'internalisation de la fonction de vente et de démarchage de la clientèle a-t-elle donc été systématisée parce qu'elle était réellement rentable ? Ou bien aussi parce que, à l'instar de certaines pratiques de management actuelles, elle a connu une vogue non directement liée à des preuves de son efficacité ? Et comment obtenir de telles preuves, puisque, même si l'on retranche du montant des contrats obtenus par le voyageur les frais liés à son déplacement, il reste difficile d'apprécier la valeur exacte des services non directement commerciaux qu'il a rendus : augmentation de la notoriété de la firme, consolidation de liens de confiance, recouvrement de créances, résolution de contentieux, etc.

\footnotetext{
${ }^{33} \mathrm{Ce}$ thème apparaît en filigrane dans presque toutes les contributions réunies ici - on pense notamment aux contributions sur la commercialisation du champagne et des soieries de B. Musset, F. Perron et F. Bayard. C'est cependant le cas de la firme Roquefort-Société, traité dans le présent numéro par S. Vabre, qui nous offre la vision la plus complète des procédures de rationalisation de la distribution qui accompagnèrent le recours croissant aux commis voyageurs.

${ }^{34}$ Ces questions sont plus particulièrement abordées dans les contributions au présent numéro de J.-P. Barrière, L. Panariti et R. Rossfeld.

${ }^{35}$ Leurs travaux sont maintenant bien connus en France grâce à l'effort de traduction consenti ces dernières années : R. H. Coase, L'entreprise, le marché et le droit, Paris, Éditions d'Organisation, 2005, O. Williamson, Les institutions de l'économie, Paris, InterEditions, 1994. On peut leur ajouter ceux de A. Greif, « Théorie des jeux et analyse historique des institutions. Les institutions économiques du Moyen Âge ", Annales HSS, 53, 3, 1998, p. $597-633$ et D. North, Le processus du développement économique, Paris, Éditions d'Organisation, 2005.

${ }^{36}$ C. F. Sabel et J. Zeitlin (eds.), Worlds of Possibilities: Flexibility and Mass Production in Western Industrialization, Cambridge, Cambridge University Press, 1997.
} 
Aussi l'étude des choix d'internalisation doit-elle prendre en compte les tâches exactes attribuées aux commis voyageurs : " internaliser » ne revient pas simplement à faire faire exactement les mêmes choses par des personnes ayant un statut juridique différent, loin de là. Il faut donc s'interroger sur les compétences professionnelles et la formation de ceux qui vendent, les modalités de leur recrutement et de leur rémunération, leur plus ou moins grande autonomie (dans le choix des itinéraires, des clients, la détermination des prix ou des conditions de vente...) et la plus ou moins grande capacité de la firme à contrôler en pratique les faits et gestes de son agent - ce qui nécessite une attention toute particulière au contexte technologique de l'époque, l'apparition du télégraphe, puis du téléphone, et plus récemment du téléphone mobile, changeant par exemple la donne. Il faut aussi considérer la stratégie adoptée par la firme : veut-elle, par exemple, plutôt vendre plus en laissant une large marge d'initiative à des agents dotés d'un fort capital relationnel et rémunérés à la commission, ou vendre bien - un produit particulièrement technique, ou fragile, ou bien encore à une clientèle particulièrement sélectionnée, plus solvable par exemple - avec des agents plus contrôlés, rémunérés essentiellement par un salaire fixe, chargés également de choyer la clientèle et responsabilisés personnellement par la prise en charge des opérations de recouvrement ${ }^{37}$ ? Plus généralement, pourquoi utilise-t-on des commis voyageurs plutôt que des dispositifs comme les catalogues ou la publicité, des correspondants sur place (lointains mais non mobiles), des déplacements de personnels non spécialisés (jeunes négociants en formation, par exemple), ou encore les services de firmes spécialisées ? Cela tient-il au type de marchés visés - opposition classique entre des marchés matures, où les produits de la firme sont déjà connus, et ceux qui restent à conquérir, entre des marchés proches et familiers et d'autres plus distants tant d'un point de vue géographique que culturel -, à la nature des produits commercialisés - qui peuvent être plus ou moins transportables, échantillonnables, standardisés... - ou encore aux conditions de vente pratiquées (formes de crédit, rapports entre producteurs, grossistes, détaillants et consommateurs...) $)^{38}$ ?

Quoi qu'il en soit, le fait que les commis voyageurs remplissent souvent de multiples fonctions est un acquis crucial du colloque de Nice - présent en réalité en filigrane dans les publications plus anciennes, mais jamais discuté pour lui-même. En effet, s'ils vendent à des détaillants et s'ils en profitent pour informer leurs employeurs de ce qu'ils perçoivent des goûts des clients et plus généralement de l'état des marchés, il leur arrive aussi de vendre aux consommateurs finaux, d'acheter des produits, d'agir plus généralement pour le compte de leur employeur et - c'est particulièrement récurrent dans les études de cas - de participer au recouvrement des créances ${ }^{39}$. Cette dernière question a fait l'objet de peu d'études historiques précises, en particulier en France, où l'image des officiers ministériels que sont les huissiers lui donne peut-être une fausse évidence : il y a en fait, en l'absence d'huissiers ou en amont de leur utilisation, bien des manières d'essayer de se faire payer, qui impliquent souvent, comme la vente, une présence sur place et des négociations verbales aussi bien qu'une connaissance fine des réputations.

\footnotetext{
${ }^{37}$ W. Friedman souligne qu'il est demandé aux commis voyageurs « to cajole the prospect » (Birth of a Salesman ..., op. cit., p. 9). Sur la question de la stratégie adoptée par l'entreprise, nous renvoyons plus précisément aux contributions de B. Musset et S. Vabre.

${ }^{38}$ Le cas du marché « atomisé » du champagne, décrit notamment par B. Musset dans sa contribution, apparaît, une fois encore, comme un remarquable terrain d'étude pour aborder ces questions. Cf. aussi, dans la contribution de J. Villain, le rôle que jouent les commis dans l'intégration de l'espace commercial lorrain et sa connexion au reste de l'Europe.

${ }^{39}$ Sur la question du recouvrement des créances, cf. notamment les contributions de B. Musset, J. Villain et A. Bartolomei.
} 
Le commis voyageur est ainsi longtemps... un commis qui voyage, c'est-à-dire quelqu'un qui se déplace pour remplir diverses tâches pour une firme, et pas seulement un vendeur. Pour comprendre l'émergence d'un métier à part, il faudrait en réalité aussi mieux connaître les autres figures d'intermédiaires qui assuraient des fonctions de représentation similaires dans l'Europe moderne, celle du fondé de pouvoir par exemple, du mandataire, du procurateur, du commissionnaire, voire du capitaine de navire marchand ${ }^{40}$, ou tout simplement de l' « agent ». Alors même que la relation «principal-agent » est un topos de la micro-économie, rares en sont les déclinaisons en études historiques vraiment précises ${ }^{41}$, que ce soit sur les conditions du contrôle et de la confiance ou sur la réalité du travail de l'agent : il y a encore beaucoup à dire sur les moyens de l'action économique à distance.

\section{La formation de la classe moyenne}

La spécialisation des tâches à laquelle on assiste, semble-t-il au $\mathrm{XX}^{\mathrm{e}}$ siècle, après une période marquée par une plus grande polyvalence, est intimement liée à la naissance d'organisations collectives revendiquant la reconnaissance de véritables professions, avec leurs statuts précis ou encore leurs formations dédiées. Les commis voyageurs n'échappent pas à ce mouvement général de professionnalisation de la fin du $\mathrm{XIX}^{\mathrm{e}}$ et du début du $\mathrm{XX}^{\mathrm{e}}$ siècle, qui les amène à délimiter un pré carré de compétences et à rechercher la distinction par rapport à des activités voisines ${ }^{42}$. Pourtant ils comptent aussi parmi les symboles d'une nouvelle classe moyenne autodidacte, apprenant sur le tas, voire ne faisant que passer dans le métier, et clairement guidée par la recherche d'une ascension sociale.

Si leur histoire sociale reste presque totalement à écrire, les quelques indices dont on dispose indiquent en effet assez que l'exercice du métier de commis voyageur n'a souvent été, dans un premier temps du moins, qu'un simple passage dans des trajectoires variées - et dès lors un point de rencontre entre des personnes d'origines diverses - plutôt qu'une véritable carrière professionnelle. Des carottages dans les sources classiques de l'histoire sociale de la France montrent l'intérêt qu'aurait une systématisation de l'étude de ces trajectoires. L'image littéraire d'un jeune homme, petit bourgeois, sans doute ni très riche ni très instruit, un Parisien qui se fait berner en province comme Gaudissart, en sort immédiatement écornée. Quinze mille actes de mariage de la région de Lille autour de 1825, 1860 et 1880, sur-représentant pourtant les zones rurales ${ }^{43}$, permettent ainsi de mesurer la croissance des mentions de l'activité (3 occurrences, puis 19, puis 40) et la transformation de sa dénomination : le premier « représentant de commerce » est mentionné en 1864, mais in fine l'expression est aussi présente que « voyageur de commerce » et plus que « commis voyageur ». Surtout, les âges sont variés, couvrant tout le spectre de 20 à 60 ans, voire occasionnellement plus, et les

\footnotetext{
${ }^{40}$ Dont Gilbert Buti, rendant compte de recherches en cours, a pu confirmer lors du colloque de Nice que nombre de ses activités, et des questions de statut et de confiance qu'elles posaient, ressemblaient à celles des commis voyageurs.

${ }^{41}$ Sur le modèle de celles menées par A. Greif, « Théorie des jeux et analyse historique des institutions », art. cit.

${ }^{42}$ Sur les luttes de frontière et les processus de professionnalisation, voir A. Abbott, The System of Professions: An Essay on the Division of Expert Labor, Chicago, The University of Chicago Press, 1988. Ces questions sont plus particulièrement abordées, dans le présent numéro, par les contributions de J.-P. Barrière, D. Andreozzi et R. Rossfeld.

${ }^{43}$ Base de données constituée pour une étude des migrations locales : cf. C. Lemercier et P.-A. Rosental, " "Pays" ruraux et découpage de l'espace : les réseaux migratoires dans la région lilloise au milieu du XIX ${ }^{\mathrm{e}}$ siècle », Population, vol. 55, $\mathrm{n}^{\circ}$ 4-5, 2000, p. 691-726. Les mentions de professions concernent les époux, leurs parents et leurs témoins.
} 
milieux sociaux tout autant. Les commis voyageurs sont loin de tous habiter à Lille ; il ne semble pas s'agir d'une profession fermée sur elle-même ou qui se transmet beaucoup (les 64 mentions sont réparties sur 62 mariages différents). Les pères des commis voyageurs peuvent être aussi bien entrepreneurs qu'ouvriers fileurs, receveurs que cabaretiers. Si leurs témoins sont assez souvent commis négociants, gardes magasins, employés de commerce ou comptables, juristes ou encore fonctionnaires, on trouve aussi un voyageur, beau-frère d'un domestique, qui épouse une journalière, aussi bien qu'un autre qui se présente comme témoin lors du mariage d'un architecte avec une professeure de musique.

Les actes notariés parisiens de 1851 confirment la variété des trajectoires qui passent par ce métier, même au moment où celui-ci commence à correspondre à un nom et à un archétype littéraire bien précis ${ }^{44}$. On y retrouve une trentaine de «commis voyageurs » et une quarantaine de "voyageurs de commerce », fils d'orfèvre ou de médecin aussi bien que de cultivateur. Parmi eux, l'époux d'une brocanteuse, le frère d'un courtier en vins (à ajouter à une liste de métiers proches ?), un "négociant et commis voyageur », ainsi que des commis voyageurs « en » librairie, produits chimiques, bonneterie, métaux ou huiles. Se spécialise-t-on ainsi occasionnellement dans une carrière de vendeur, ou s'agit-il d'une véritable expertise pouvant conduire à rester dans un secteur en changeant de fonction ? À partir de dossiers de faillites, une étude locale du secteur de la chaussure montre que d'anciens représentants deviennent associés de fabricants, tout en se mariant souvent dans leur famille ${ }^{45}$.

Le commis voyageur d'ailleurs, Walter Friedman le souligne, emprunte nombre de ses pratiques quotidiennes à d'autres itinérants, charlatans ou prêcheurs, colporteurs ou marchands ambulants : au-delà d'une analogie, y a-t-il ici des liens directs, des passages d'une activité à l'autre ? En Oisans, des colporteurs sont ainsi devenus graduellement commis voyageurs pour des épiciers, passant d'une clientèle rurale à une clientèle urbaine de détaillants et à la vente sur échantillons. Mais d'autres colporteurs plus importants, à la fin du XIX $\mathrm{X}^{\mathrm{e}}$ siècle, recevaient eux-mêmes, l'été, la visite de voyageurs de commerce envoyés par leurs fournisseurs : la hiérarchie sociale entre les deux activités ne semble donc pas donnée d'avance ${ }^{46}$. En revanche, elle est hautement revendiquée par les commis voyageurs lorsqu'ils commencent à s'organiser collectivement, à un moment, il est vrai, où l'ambulance est particulièrement contrôlée et stigmatisée en Europe ${ }^{47}$. Eux-mêmes soumis à l'évolution des documents d'identification des voyageurs, les représentants de commerce défendent leur supériorité par rapport aux colporteurs et marchands ambulants ; aux États-Unis, le voyageur de commerce s'affirme aussi comme un WASP, alors que les Juifs, mais aussi les femmes, sont très présents dans le colportage $^{48}$.

\footnotetext{
${ }^{44}$ Bases ARNO interrogeables sur http://www.archivesnationales.culture.gouv.fr/arn/ (consultation du 12 juin 2010 ).

${ }^{45}$ J. Cucarull, « L'industrie de la chaussure à Fougères avant 1914 d'après les dossiers de faillite des entreprises ", Annales de Bretagne et des Pays de l'Ouest, 114, janvier-mars 2007, mis en ligne le 30 mars 2009. Consulté le 11 octobre 2010 . URL : http://abpo.revues.org/602

${ }^{46} \mathrm{Ch}$. Robert-Muller et A. Allix, « Un type d'émigration alpine : les colporteurs de l'Oisans », Revue de géographie alpine, 11, 1923, p. 585-634.

${ }^{47}$ C. Zalc, « Contrôler et surveiller le commerce migrant. Nomades, forains et ambulants à Paris (1912-1940) », in M.-C. Blanc-Chaléard, C. Douki, N. Dyonet et V. Milliot (dir.), La police et les migrants, Rennes, Presses universitaires de Rennes, 2001, p. 365-388.

${ }^{48}$ W. Friedman, Birth of a Salesman ..., op. cit., p. 60.
} 
Cette volonté de se situer dans les parties supérieures de la classe moyenne et de se différencier notamment des plus petits indépendants a pour partie abouti en termes de statut juridique, comme le montrent certaines des contributions publiées ici $^{49}$. Il faut certes attendre l'évolution de la nomenclature INSEE des professions et catégories sociales en 1984 pour que les « voyageurs de commerce » quittent la catégorie « employés de commerce » pour rejoindre les « représentants de commerce » (anciens « cadres moyens ») parmi les « professions intermédiaires ». Mais, en pratique, ces métiers ont une liberté et une amplitude d'horaires de travail, par exemple, qui les place à la fin du $\mathrm{XX}^{\mathrm{e}}$ siècle dans la moyenne des cadres ${ }^{50}$. Qu'en est-il de leurs revenus et de leur reconnaissance sociale ? La réponse est sans doute moins claire, même si une frange supérieure proche du monde du négoce paraît s'être toujours maintenue. Les voyageurs étudiés par Gérard Gayot étaient ainsi les mieux payés des employés de commerce, et il y avait quelques représentants de commerce sur les listes de « notables commerçants » du Second Empire ${ }^{51}$.

Cette affirmation d'un métier de représentant de commerce, là où il n'y avait initialement qu'un rôle tenu transitoirement et parmi d'autres, nous ramène finalement à la question de l'implication des représentants dans la dépersonnalisation supposée des relations économiques : sont-ils les simples porteurs interchangeables d'un costume préétabli ou bien de véritables personnes, pour ne pas dire des personnalités, susceptibles non seulement d'incarner l'entreprise, mais encore d'influencer les clients dans un colloque singulier?

\section{Un commerce désencastré ?}

L'histoire des commis voyageurs permet en effet de discuter de notions souvent mises en avant, mais pas toujours précisément problématisées : l'informel et le formel, le personnel et l'impersonnel, ou encore, pour reprendre la célèbre proposition formulée par Mark Granovetter, la question de la « force des liens faibles » ${ }^{52}$. Si les définitions de toutes ces catégories sont loin de faire consensus, on peut facilement considérer que le recours à des commis voyageurs marque le recul de la personnalisation et le triomphe des stratégies fondées sur l'activation de liens faibles. Certes, l'historiographie de la période moderne tend aujourd'hui à relativiser le poids des liens familiaux ou communautaires dans l'activité économique et à insister sur l'existence d'un important commerce à distance liant des marchands de langues et de religions différentes ${ }^{53}$. Il reste que ce commerce se fonde bien sur des réputations, personnelles ou familiales, entretenues par des formes de correspondance ou encore d'amitié ritualisée.

\footnotetext{
${ }^{49}$ Cf. les articles de J.-P. Barrière, D. Andreozzi et R. Rossfeld.

${ }^{50}$ A. Chenu, « Les horaires et l'organisation du temps de travail », Économie et statistique, n 352, 2002, p. 151-167.

${ }^{51}$ J.-C. Martin, « Hiérarchie et structure de la société commerçante : les listes d'électeurs au tribunal de commerce de Niort en 1864 et 1874 », Le Mouvement Social, juillet-septembre 1980, p. 57-77, notamment p. 66.

${ }^{52}$ M. S. Granovetter, « The Strength of Weak Ties », American Journal of Sociology, 78, 6, 1973, p. 1360-1380, traduit dans Le Marché autrement. Essais de Mark Granovetter, Paris, Desclée de Brouwer, 2000.

${ }^{53}$ Voir par exemple l'article de F. Trivellato, « Juifs de Livourne, Italiens de Lisbonne, hindous de Goa. Réseaux marchands et échanges interculturels à l'époque moderne ", Annales HSS, n 3, 2003, p. 581-603, repris et développé dans son ouvrage le plus récent (The Familiarity of Strangers : The Sephardic Diaspora, Livorno, and Cross-Cultural Trade in the Early Modern Period, New Haven, Yale University Press, 2009) et G. Lydon, On Trans-Saharan Trails: Islamic Law, Trade Networks and Cross-Cultural Exchange in Nineteenth-Century Western Africa, Cambridge, Cambridge University Press, 2009.
} 
Par rapport à de telles relations directes entre négociants fondées sur la réputation ou sur des liens sociaux préexistants, la naissance du commis voyageur implique le recours à un agent subordonné qui peut être extérieur au groupe, voire seulement de passage dans cette fonction, qui est souvent totalement inconnu, à titre personnel du moins, des clients qu'il visite, et qui doit - plus ou moins - disparaître derrière les consignes qui lui ont été fixées, s'en tenir à l'itinéraire qu'on lui a indiqué, présenter un catalogue pré-imprimé ou suivre des consignes strictes sur les modalités de paiement. Cependant le commis voyageur n'est pas seulement cet anonyme qui transporterait des échantillons au prix déjà fixé. Il doit gagner ou conserver la confiance de l'acheteur et informer sa firme sur le crédit de ses partenaires : les questions de réputation restent donc très importantes, même lorsque l'information à leur sujet passe par des agents ${ }^{54}$.

Plus fondamentalement, Walter Friedman considère que l'emploi de représentants de commerce a eu précisément pour but de conférer à des relations entre fournisseur et client à tout point de vue « faibles », car anonymes et dépersonnalisées, la force de liens forts : la chaleur du contact et la confiance qui s'établit au cours du bref échange (la " poignée de main visible » du commis voyageur venant appuyer la main invisible du marché ${ }^{55}$ ) remplaceraient l'appui sur un dispositif plus classique fondé sur la recommandation ou la réputation. Il remarque aussi que le drummers' power déclina lorsque les détaillants ne souhaitèrent plus acquérir les produits auprès d'un grossiste dont ils connaissaient personnellement le représentant, mais plutôt auprès d'un fabricant, dont ils connaissaient les produits ou la marque à travers la presse et la publicité ${ }^{56}$. Au court-circuitage de circuits traditionnels du négoce par les commis voyageurs aurait fait suite, plus tard, celui des représentants de commerce par la publicité et le marketing. La concurrence entre les deux métiers est de fait immédiatement exacerbée. Ainsi, l'agence Havas présente en 1935 l'annonce de presse comme « un représentant qui visite chaque jour des milliers de clients [...], bien introduit et pas cher $»^{57}$.

Le voyageur de commerce incarne-t-il alors une période de transition entre un monde de liens forts et l'univers supposé totalement impersonnel de la vente en ligne, par exemple ? La question est en réalité plus complexe puisque, loin d'avoir disparu, la sphère de la représentation commerciale n'a au contraire cessé de s'étoffer au cours du $\mathrm{XX}^{\mathrm{e}}$ siècle et encore dans les dernières décennies, pourtant contemporaines de la révolution numérique ${ }^{58}$. Mieux comprendre sa naissance et plus encore sa survie permettrait donc de nuancer cette vision linéaire et de mettre plutôt l'accent sur les activités humaines et les auxiliaires matériels qui permettent de mêler le personnel et l'impersonnel, pour le plus grand bien des acteurs économiques qui y parviennent ${ }^{59}$. Les « voyageurs intelligents » du XVIII ${ }^{\mathrm{e}}$ siècle sont déjà munis, répétonsle, de lettres circulaires les introduisant auprès des clients : un bel exemple d'utilisation de l'impersonnel au service du personnel.

\footnotetext{
${ }^{54}$ Ces questions sont plus précisément abordées, dans le présent numéro, dans l'article d'A. Bartolomei.

${ }^{55}$ W. A. Friedman, Birth of a Salesman ..., op. cit., p. 7.

${ }^{56}$ Ibid., p. 87.

${ }^{57}$ M. Martin, « Structures de société et consciences rebelles : les résistances à la publicité dans l'entre-deux-guerres », Le Mouvement Social, $\mathrm{n}^{\circ}$ 146, juillet-septembre 1989, p. 27-48, citation p. 40 ; M.-E. Chessel, La publicité. Naissance d'une profession (1900-1940), Paris, CNRS Éditions, 1998.

${ }^{58}$ Sur ce point, nous renvoyons à l'entretien avec Jacques Delétang et Nadine Autréau, président et conseillère juridique de la Fédération Nationale des Agents de Commerce, réalisé par A. Bartolomei et C. Lemercier dans le présent numéro de la revue Entreprises et Histoire.

${ }^{59} \mathrm{Ce}$ qui rejoint les intuitions générales de J.-P. Hirsch, Les deux rêves du commerce : entreprise et institution dans la région lilloise, 1780-1860, Paris, Editions de l'EHESS, 1991.
} 